\title{
REVIEW
}

\section{Clinical review: Biomarkers of acute kidney injury: where are we now?}

Marlies Ostermann 1,*, Barbara J Philips ${ }^{2}$ and Lui G Forni ${ }^{3}$

\begin{abstract}
The recognition that acute kidney injury (AKI) is a significant independent risk factor for morbidity and mortality has resulted in a substantial number of publications over the past 5 years or more. In no small part these have, to a degree, highlighted the inadequacy of conventional markers of renal insufficiency in the acute setting. Much effort has been invested in the identification of early, specific AKI markers in order to aid early diagnosis of AKI and hopefully improve outcome. The search for a 'biomarker' of AKI has seen early promise replaced by a degree of pessimism due to the lack of a clear candidate molecule and variability of results. We outline the major studies described to date as well as discuss potential reasons for the discrepancies observed and suggest that evolution of the field may result in success with ultimately an improvement in patient outcomes.
\end{abstract}

\section{Introduction}

Historically, managing patients with acute renal failure suffered, in part, from a lack of consensus regarding definition. This has been addressed, to a degree, with the introduction of diagnostic criteria based on changes in both serum creatinine and urine output. The RIFLE (Risk - Injury - Failure - Loss - End stage renal disease) criteria were developed in order to describe patients in terms of conventional markers of renal function, allowing cohort selection for studying intervention [1]. Hence, the term acute kidney injury (AKI) has gained popularity, although these criteria are not diagnostic and do not confer a cause for AKI but instead alert the clinician to the occurrence of biochemical and physiological changes in keeping with renal insufficiency. The RIFLE criteria

*Correspondence: Marlies.Ostermann@gstt.nhs.uk

'King's College London, Guy's and St Thomas' Hospital, Department of Critical Care and Nephrology, London SE1 7EH, UK

Full list of author information is available at the end of the article were subsequently modified by the AKI Network (AKIN) group [2]. Numerous publications have 'validated' the RIFLE and AKIN classifications in terms of patient outcome prominently in the ICU arena. Interest into patients with AKI in the United Kingdom was galvanized following the publication of the National Confidential Enquiry into Patient Outcomes and Death (NCEPOD) report Adding Insult to Injury in 2009, which highlighted inadequacies in care for up to $50 \%$ of patients with AKI predominantly admitted to general wards [3].

In everyday practice, clinicians presented with a patient with a raised serum creatinine face several clinical challenges including: differentiation between pre-renal azotaemia, AKI and stable chronic kidney disease (CKD); estimation as to the degree of renal dysfunction compared to baseline; estimation of the chances of renal recovery and need for renal replacement therapy (RRT); and decisions with regard to any specific management

In 2005, the American Society of Nephrology Renal Research Report assigned the highest research priority to the standardization and/or discovery of new biomarkers of AKI driven by the awareness that even relatively 'trivial' increases in serum creatinine were associated with considerable increases in the utilisation of health resources [4]. Since then, we have seen a proliferation of studies focussing on the detection and validation of new biomarkers in a variety of different patient populations and clinical settings.

Any potentially clinically useful candidate AKI biomarker(s) should ideally fulfil several criteria, as outlined in Table 1. When one examines the performance of the existing AKI biomarkers against these criteria the results are variable, and their diagnostic and prognostic value in clinical practice outside a well defined research setting is somewhat unreliable. The purpose of this review is to summarize recent findings concerning biomarkers for AKI in adult patient populations, including patients in the ICU, to highlight their strengths and weaknesses, and to determine their usefulness in clinical practice.

\section{Different biomarkers evaluated in clinical trials}

Recently identified biomarkers of AKI are either low molecular weight proteins that are present in the 
To provide information above that of traditional clinical evaluation and investigation

To be non-invasive, utilising easily accessible samples

To provide results rapidly and both sensitive and specific to AKI

To have specific cutoff values to distinguish between normal and abnormal renal function

To distinguish intrinsic AKI from pre-renal azotaemia

To provide insight into aetiology of AKI

To differentiate between $\mathrm{AKI}$ and chronic kidney disease

To be specific for renal injury in the presence of concomitant dysfunction of other organs

To be indicative of the severity of AKI

To ideally allow some estimate as to the timing of the onset of renal injury

To guide initiation of therapies and to monitor the response to interventions

To aid prognostication in terms of potential renal recovery, need for RRT and mortality

AKI, acute kidney injury; RRT, renal replacement therapy.

systemic circulation and undergo glomerular filtration (that is, markers of glomerular function), enzymes that are released by tubular cells into the urine after tubular cell injury (that is, markers of tubular damage) or inflammatory mediators released by renal cells or infiltrating inflammatory cells (that is, markers of degree of damage and indicators of site of injury) (Figure 1 and Table 2). The aetiology of AKI is far from uniform, however, and defining new biomarkers for AKI is thus extremely challenging. Consequently, investigators have tended to study their chosen biomarker(s) in well defined clinical settings where the timing of renal injury is known, that is, after cardiopulmonary bypass surgery, coronary angiography or following renal transplantation (Table 3). Furthermore, a significant number of studies were done in paediatric populations where comorbidities such as CKD, diabetes mellitus and chronic inflammatory diseases are less likely to present as confounding variables. As a result, not all studies are easily generalized to heterogenous populations, including critically ill patients in the ICU. The effects of baseline renal function, comorbidities, age, and duration of renal injury have led to conflicting results in various studies (Table 3 ).

\section{Specific clinical scenarios \\ Differentiation between 'pre-renal' and 'intrinsic' acute kidney injury}

The differentiation between a transient serum creatinine rise caused by perturbations in renal perfusion and direct damage to the kidney leading to sustained AKI can be difficult, especially in acute patients. The diagnosis of pre-renal AKI is usually retrospective after a transient rise in serum creatinine with recovery of function within 24 to 72 hours. In a study involving 510 critically ill patients, De Geus and colleagues [5] confirmed that serial measurement of urinary neutrophil gelatinase-associated lipocalin (NGAL) could distinguish between these two

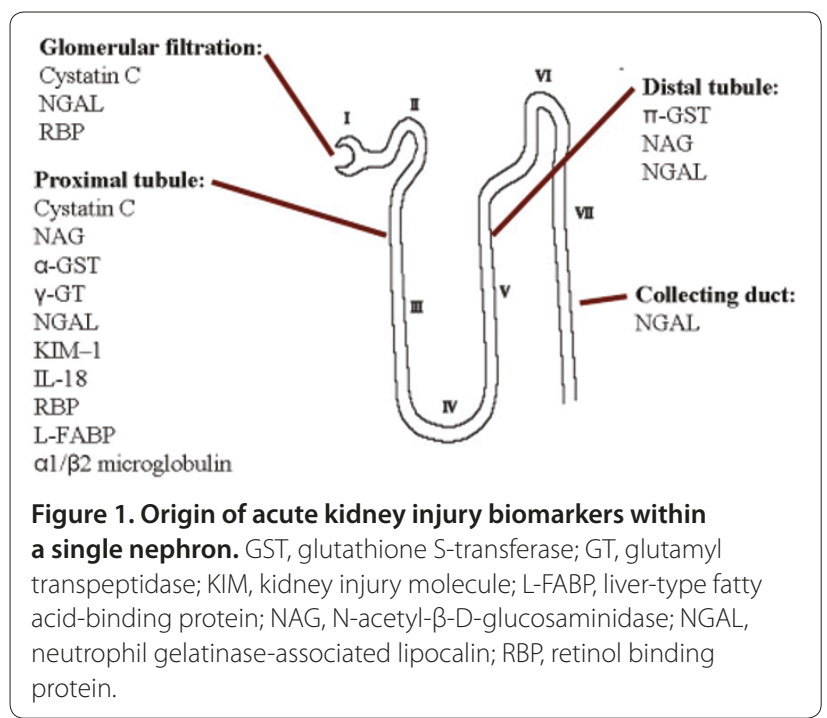

conditions. Adding the results to a clinical prediction model, however, only led to marginal improvement of the area under the receiver operating characteristics curve (AUC) from 0.79 to 0.82 . Nickolas and colleagues [6] evaluated five different urinary biomarkers (NGAL, kidney injury molecule (KIM)-1, liver-type fatty acidbinding protein (L-FABP), IL-18, and cystatin C) in 1,635 patients who presented to the emergency department and were subsequently hospitalized for more than 24 hours. The entire cohort was divided into three subgroups: patients with sustained AKI (that is, AKI that persisted for more than 72 hours); patients with transient AKI (that is, AKI that resolved within 72 hours); and patients without AKI. All markers were raised in patients with sustained AKI but only urinary NGAL (uNGAL) and urinary cystatin $C$ were able to distinguish patients with sustained AKI from those with transient or prerenal AKI. Hall and colleagues [7] measured UNGAL, 


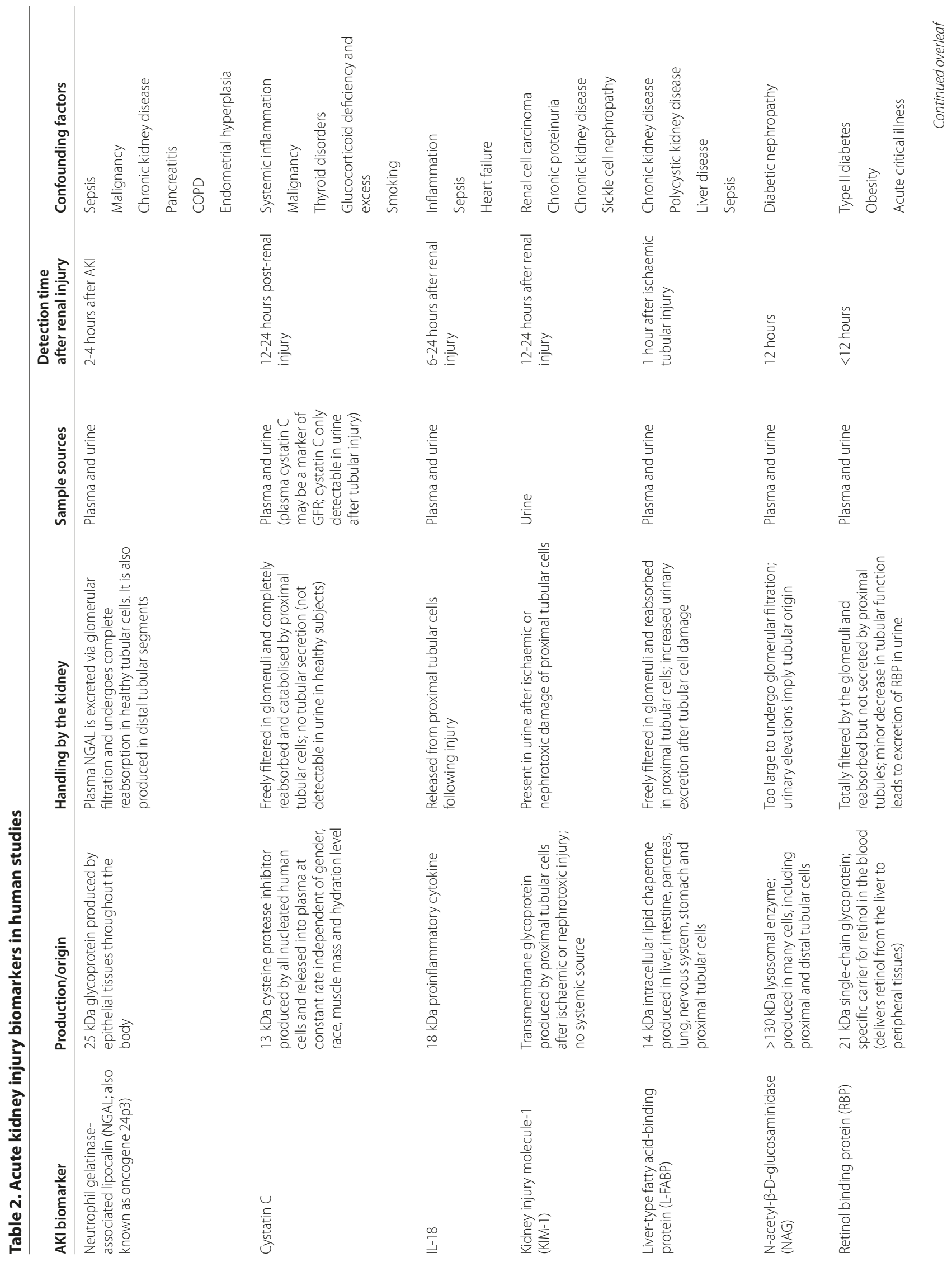




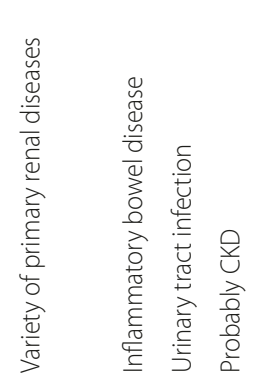

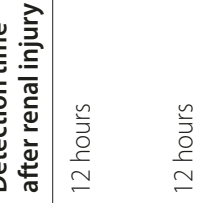

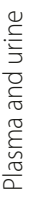
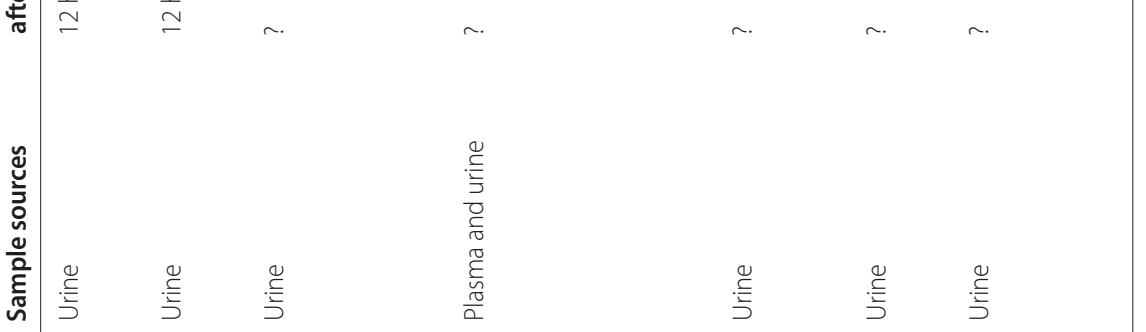

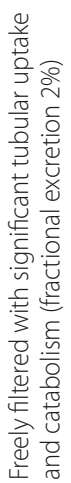
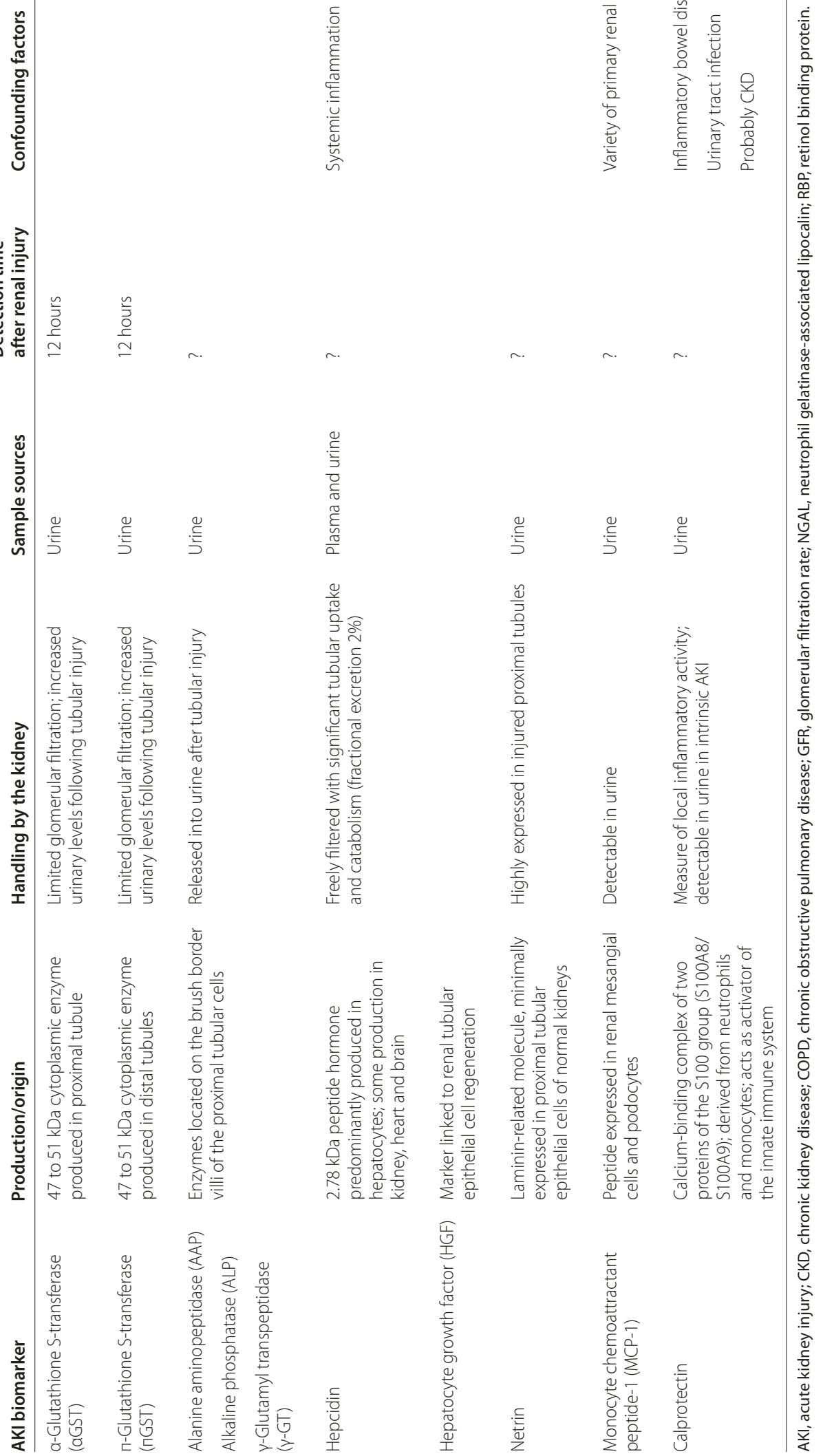
Table 3. Clinical studies of common acute kidney injury biomarkers in adult patients

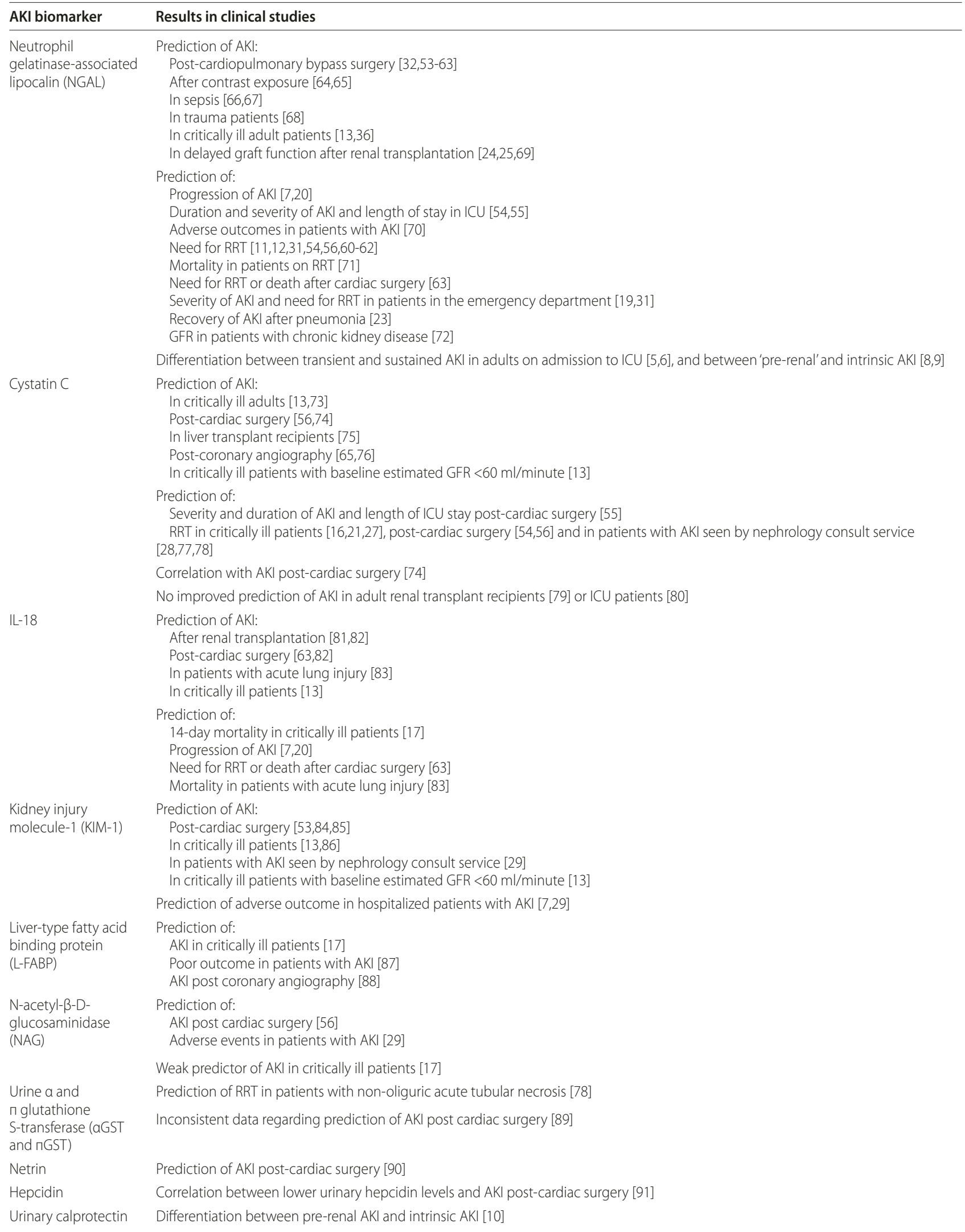

AKI, acute kidney injury; GFR, glomerular filtration rate; RRT, renal replacement therapy. 
KIM-1 and IL-18 in 249 hospitalised adult patients on the first day when AKIN criteria for AKI were met. They found that the AUC for prediction of progression to a higher AKIN stage or hospital death was 0.62 using a clinical model including baseline glomerular filtration rate (GFR), surgery, diabetes mellitus, hypertension, age $\geq 65$ years, body mass index, male gender and nonCaucasian race. The individual addition of NGAL, KIM-1 and IL-18 to the clinical model improved the AUC to $0.75,0.69$ and 0.68 , respectively. Nejat and colleagues [8] measured uNGAL, cystatin $C$, $\gamma$-glutamyl transpeptidase ( $\gamma$-GT), IL-18 and KIM-1 in 529 critically ill patients at 0 , 12 and 24 hours after admission to ICU. Patients were stratified into groups having no AKI, pre-renal AKI, AKI with recovery within 24 to 48 hours and patients with the composite of AKI for $>48$ hours and/or treatment with RRT. Biomarker concentrations significantly and progressively increased with duration of AKI. In patients defined as having pre-renal AKI, urinary cystatin C, IL-18 and KIM-1 levels were significantly higher compared to patients without AKI, and significantly lower when compared to patients with AKI for $>48$ hours or patients needing RRT. There was no significant difference for uNGAL and $\gamma$-GT levels between patients with pre-renal AKI versus no AKI. Singer and colleagues [9] explored the use of UNGAL in differentiating between pre-renal and intrinsic AKI in patients with a raised serum creatinine. On adjudication by two clinicians, $26 \%$ of the patients were labelled as unclassifiable as their clinical and laboratory parameters did not conform to traditional pre-renal or AKI criteria. While the NGAL results generally tracked the patients who were classified as having pre-renal or AKI, disappointingly there was considerable overlap of NGAL values in the unclassifiable group. Finally, Heller and colleagues [10] recently explored the role of urinary calprotectin, a mediator protein of the innate immune system, in differentiating between prerenal AKI and intrinsic AKI. A cohort of 101 hospitalised patients was studied, including 34 patients with pre-renal AKI and 52 patients with intrinsic AKI of whom over $40 \%$ had a urinary tract infection. The median urinary calprotectin was significantly higher in intrinsic AKI than in pre-renal AKI. Importantly, there was no significant difference between healthy controls and patients with pre-renal AKI. The AUC for urinary calprotectin for predicting intrinsic AKI was 0.97.

In conclusion, given the wide variation between studies, no single biomarker can currently be recommended to differentiate reliably between pre-renal AKI and intrinsic AKI.

\section{'Early' diagnosis of acute kidney injury}

Many investigations have focussed on the ability of biomarkers to diagnose AKI before a detectable serum creatinine rise. Cruz and colleagues [11] measured plasma NGAL (pNGAL) in 301 heterogenous ICU patients and showed that it allowed the diagnosis of AKI up to 48 hours prior to AKI according to the RIFLE criteria with an AUC of 0.78. Of note, there was also a strong association between pNGAL and overall disease severity regardless of the presence of AKI. In another study involving 88 patients, a single pNGAL measurement on admission to ICU predicted the onset of AKI 48 hours before AKI as per RIFLE criteria with an impressive AUC of 0.92 [12]; however, the study was small and patients with a history of CKD were excluded. In contrast, Endre and colleagues [13] evaluated 6 urinary biomarkers (NGAL, KIM-1, IL-18, alkaline phosphatase, $\gamma$-GT and cystatin C) prospectively in 529 patients on admission to ICU and found that no biomarker had an AUC above 0.7 in the prediction of AKI, RRT or mortality. De Geus and colleagues [14] assessed the ability of pNGAL and uNGAL to predict severe AKI prospectively in a cohort of 632 consecutive adult critically ill patients. Samples for measurement of NGAL were obtained on admission and thereafter at 4, 8, 24, 36 and 72 hours. A total of 171 (27\%) patients developed AKI according to the RIFLE classification during the first week of ICU stay. There was a significant association between patients' pNGAL and UNGAL levels on ICU admission and the final RIFLE class. Combining both biomarkers with 13 clinical and laboratory parameters in a logistic regression model improved the prediction for RIFLE Failure. However, there was no validation cohort. Also, $29.8 \%$ of patients with sepsis had elevated uNGAL levels without AKI as defined by the RIFLE criteria.

Several studies have demonstrated that measurement of plasma cystatin $C$ concentrations may be a useful tool to diagnose AKI early but its superiority over serum creatinine has not been universally demonstrated. A recent meta-analysis by Zhang and colleagues [15] included 13 studies and found that the AUC of plasma cystatin C to predict AKI was between 0.86 to 0.96 depending on the inclusion criteria employed. In contrast, urinary cystatin C levels had only moderate diagnostic value, with a pooled AUC of 0.64 (95\% confidence interval (CI) 0.62 to 0.66 ). The conclusions of the meta-analysis were limited by great heterogeneity of pooled studies, in terms of clinical setting, definition of AKI, timing of plasma/urinary cystatin C measurement and type of assay used. Royakkers and colleagues [16] measured serial plasma and urinary cystatin C prospectively in 151 ICU patients and assessed the performance for AKI prediction on day 1 and day 2 before the RIFLE criteria for AKI were met. On day 2, plasma and urinary cystatin $\mathrm{C}$ had an AUC for predicting AKI of 0.72 and 0.49 , respectively. On day 1 , the AUCs were respectively 0.62 and 0.46 . Of note, plasma cystatin $C$ levels did not rise earlier than serum creatinine. 
Doi and colleagues [17] measured 5 different urinary biomarkers (L-FABP, NGAL, cystatin C, IL-18 and albumin) in 339 critically ill adult patients on admission to a medical-surgical ICU of whom 131 developed AKI as defined by the RIFLE criteria. In this cohort, the best urinary biomarker to detect AKI was L-FABP with an AUC of 0.75 (95\% CI 0.69 to 0.80 ).

Outside the ICU, the predictive potential of pNGAL for AKI, compared to serum creatinine, was assessed in 207 consecutive patients presenting to the emergency department with acute heart failure [18]. pNGAL was measured in a blinded fashion at presentation and serially thereafter. Sixty patients (29\%) developed AKI according to the AKIN criteria. After control for pre-existing chronic cardiac or kidney disease in a multivariate regression analysis, serum creatinine, but not NGAL, remained an independent predictor of AKI (hazard ratio 1.15; 95\% CI 1.01 to $1.31 ; P=0.04)$. In a separate study including emergency patients with suspected sepsis at risk of AKI, Shapiro and colleagues [19] found that a cutoff pNGAL value of $>150 \mathrm{ng} / \mathrm{ml}$ demonstrated a sensitivity of $81 \%$ for predicting RIFLE Risk and 93\% for prediction of RIFLE Injury; however, specificity was poor at 51\% (95\% CI 47 to $55 \%$ ).

In conclusion, the diagnostic utility of NGAL or other biomarkers to diagnose AKI prior to elevations in creatinine varies between different patient populations and is affected by comorbidity, timing of the measurements and, importantly, the chosen cutoff values. Herein lies one of the fundamental problems besetting the evaluation and interpretation of any new biomarker for AKI. At present the best routinely applied test for renal function and presumed GFR is that of serum creatinine. This is far from a robust gold standard and is beset with problems in the acute setting and in particular in the ICU patient with inherent problems associated with creatine production rates as well as fluctuating volumes of distribution.

\section{Prediction of outcome}

Different outcomes have been evaluated in clinical studies, including severity and progression of AKI, need for RRT, ICU and hospital mortality, and progression to CKD. In a prospective multicenter observational cohort study of 380 adults with confirmed AKI stage 1 as per AKIN criteria after cardiac surgery, Koyner and colleagues [20] evaluated the role of urinary IL-18, urinary albumin to creatinine ratio (ACR), and UNGAL and PNGAL in predicting risk of progression of AKI. After adjustment for clinical predictors, an ACR $>133 \mathrm{mg} / \mathrm{g}$ at the time of AKI denoted 3.4-fold odds of AKI progression compared with an ACR $<35 \mathrm{mg} / \mathrm{g}$, and a urine IL-18 level $>185$ showed a three-fold risk of progression compared with lower levels. uNGAL levels were not statistically significant after adjustment of clinical variables. A pNGAL level $>323 \mathrm{ng} / \mathrm{ml}$ performed the best, conveying an over seven-fold risk of AKI progression compared to patients with lower values.

Mortality is a common outcome in several biomarker studies. Bell and colleagues [21] demonstrated that among a mixed ICU population, plasma cystatin $C$ levels on admission correlated with mortality both in ICU patients with or without AKI. Doi and colleagues [17] showed that a combination of uNGAL, urinary cystatin C and IL-18 predicted 14-day mortality with an impressive AUC of 0.93 (95\% CI 0.95 to 0.94). In contrast, Nejat and colleagues [22] reported that plasma cystatin C levels on ICU admission were predictive of death within 30 days but the observed AUC was only 0.63 (95\% CI 0.56 to 0.72). Thus, plasma cystatin $C$ appears to be a good biomarker to estimate GFR and to detect any deterioration earlier than serum creatinine but its ability to reliably predict AKI, RRT requirement or ICU/hospital death remains uncertain.

Recovery of renal function is another important clinical outcome. The ability of pNGAL to predict the recovery from AKI was demonstrated in a post hoc analysis of a multicenter, prospective, cohort study of 180 patients with community-acquired pneumonia and AKI (defined as RIFLE Failure) [23]. pNGAL alone predicted failure to recover from AKI with an AUC of 0.74 (95\% CI 0.66 to 0.81 ), similar to a clinical model using age, serum creatinine, pneumonia severity, and non-renal organ failure with an AUC of 0.78. Importantly, combining the clinical model with pNGAL concentrations did not improve predictive ability.

uNGAL and pNGAL have also been studied as predictors of graft function after renal transplantation. Hollmen and colleagues [24] measured NGAL values in 99 consecutive deceased kidney donors in the ICU (176 recipients) and found that increased donor UNGAL level but not pNGAL predicted an increase in observed histological changes in subsequent donor kidney biopsies, an increase in delayed graft function beyond 14 days and worse 1-year graft survival. In a smaller prospective study, pNGAL was evaluated in a cohort of 41 deceased kidney donors, and in contrast to the work of Hollmen and colleagues, pNGAL was superior to serum creatinine in predicting delayed graft function [25].

\section{Triggers for renal replacement therapy}

As discussed, some AKI biomarkers have the capacity, either alone or integrated with traditional renal function tests, to predict the need for RRT in a variety of clinical settings [26] (Table 3). Herget-Rosenthal and colleagues [27] measured plasma cystatin C daily in 85 patients (44 patients with AKI and 41 controls) in 3 German medical and surgical ICUs and showed that an increase by $\geq 50 \%$ 
predicted the requirement of RRT with AUCs of 0.69 ( $95 \%$ CI 0.51 to 0.84 ) when measured 2 days prior to developing AKI as per RIFLE criteria, 0.75 (95\% CI 0.62 to 0.85 ) one day prior to $\mathrm{AKI}$, and 0.76 (95\% CI 0.62 to 0.89 ) on the day of RIFLE Risk. Of note, the precise indications for RRT were not provided. In contrast, Perianayagam and colleagues [28] measured plasma cystatin C levels in 200 hospitalised patients with AKI at the time of nephrology consultation and showed that cystatin $C$ performed no better than traditional indices of kidney injury (blood urea nitrogen, serum creatinine and urine output) in predicting RRT requirement or inhospital death (AUC 0.65 ; $95 \%$ CI 0.58 to 0.73 ). Similarly, in the previously cited study by Royakkers and colleagues [16], plasma and urine cystatin $C$ at the onset of AKI were poor predictors for the need of RRT, with AUCs of 0.66 and 0.61 , respectively. Liangos and colleagues [29] measured urinary KIM-1 and N-acetyl- $\beta$-D-glucosaminidase in 201 hospitalised patients with AKI and found that both biomarkers in combination with cirrhosis, sepsis, oliguria and mechanical ventilation yielded an AUC of 0.78 (95\% CI 0.71 to 0.84 ) in predicting need for RRT or mortality. However, one wonders whether clinical evaluation alone would not predict this outcome equally well.

Although data confirm that a higher biomarker level is often associated with RRT, there is still insufficient evidence to conclude that biomarkers can be used routinely to decide when to initiate RRT. In clinical practice, the decision to start RRT is usually influenced not only by serum creatinine but by multiple factors, including individual physician practice, degree of azotaemia, acidosis and fluid overload and severity of illness of the patient as well as organisational or logistical factors. Future studies will have to explore whether specific biomarkers can indeed identify AKI that is so severe that spontaneous recovery is unlikely to occur and RRT is needed, and whether they can be integrated into clinical RRT decision algorithms.

\section{Applicability of new AKI biomarkers in clinical practice}

The performance of different urinary and plasma biomarkers for AKI has been very variable with AUCs quoted between 0.3 and 1.0 [30]. Studies with higher AUC values often included homogenous populations with a well-defined single injury to the kidney, such as those patients receiving contrast, after cardiac surgery or following transplantation. In more heterogenous populations including critically ill patients in the ICU or emergency department where time of onset of renal injury is variable, the biomarkers tend to perform less well with lower derived AUC values.

The time at which biomarker levels are measured clearly influences their test performance. With the rapid changes in pNGAL and uNGAL concentrations, the slow changes in serum creatinine, and the effects of intensive resuscitation and fluid administration in the first hours after ICU admission, timing of NGAL measurement in relation to serum creatinine affects interpretation. In previous studies in ICU patients, NGAL measurement ranged from 48 hours after the initiation of mechanical ventilation to within 24 hours of admission to the initial presentation in the ICU. In the aforementioned paper by De Geus and colleagues [14], 58\% of all patients with AKI already had obvious AKI on admission to ICU. Similarly, Nickolas and colleagues [31] reported that a uNGAL level $>85 \mu \mathrm{g} / \mathrm{g}$ creatinine was an excellent predictor of AKI in the emergency department setting with an AUC of 0.95 . However, the mean serum creatinine of the entire AKI subgroup at presentation in the emergency department was $495 \mu \mathrm{mol} / \mathrm{L}$ (standard deviation 486), indicating that severe loss of renal function had already occurred, and was apparent, in most patients. Ideally, test results generated in patients with established AKI should not be used for the comparison with those in a cohort at risk of developing AKI.

Recent studies in healthy volunteers have also emphasized that there may be gender-related differences (that is, higher NGAL levels in women) and age-related variations in biomarker levels [32]. This uncertainty about the exact cutoff values to differentiate normal renal function from AKI, coupled with poor performance in patients with pre-existing CKD or other comorbidities, has significantly limited the utility of AKI biomarkers [13,33]. In a cohort of patients following cardiopulmonary bypass surgery, McIlroy and colleagues [33] showed that uNGAL was only predictive of AKI in patients with normal renal function before surgery but not in patients with CKD. Finally, there is an ongoing debate about the interpretation of urinary biomarkers and whether it is necessary to normalize the values to urinary creatinine $[34,35]$.

An ideal biomarker should provide additional information that is not surmised from clinical evaluation and traditional tests. Although numerous studies have confirmed that various biomarkers indeed predict AKI earlier than serum creatinine alone, their superiority over clinical models is uncertain. The danger is that indiscriminate use of biomarkers may distract from adequate clinical evaluation and result in worse patient outcomes as well as consuming significant resources. Siew and colleagues [36] showed that NGAL added little to clinical prediction in discriminating AKI in a general ICU population. De Geus and colleagues [5] confirmed that serial UNGAL results were superior to pNGAL and cystatin $\mathrm{C}$ in differentiating between transient and sustained AKI on admission to ICU but adding UNGAL to a prediction model with clinical parameters improved the model only marginally. Singer and colleagues [9] 
showed that NGAL results generally tracked the patients classified as having pre-renal or AKI but there was considerable overlap of NGAL values in the unclassifiable group. This inability of NGAL to improve the classification of the unclassifiable patients, in whom it would have the most value, illustrates some of the gaps in our current knowledge.

Finally, some studies have shown that the predictive property of various biomarkers improves with increased frequency of measurements. However, whether frequent measurements several times a day are practical and costeffective in routine clinical practice has not been evaluated.

\section{Traditional markers of renal function}

Current tools to diagnose AKI and to distinguish intrinsic AKI from pre-renal AKI include blood urea nitrogen, urine output and urine chemistry and microscopy.

\section{Urea}

Urea is formed by the hepatic metabolism of amino acids and excreted primarily by glomerular filtration. Serum concentrations may vary as a result of changes in urea production (that is, in the context of a gastrointestinal bleed) and tubular urea reabsorption during hypovolaemic states without changes in GFR. This makes urea an unreliable marker of renal function.

\section{Fractional excretion of sodium}

Fractional excretion of sodium $(\mathrm{FeNa})$ was one of the first urine chemistries applied to differentiating pre-renal AKI from acute tubular necrosis (ATN). It is based on the premise that intact tubules reabsorb sodium in the prerenal setting whereas injured tubules in the context of ATN do not. In one of the earlier studies from 1976, Espinel [37] showed in 17 highly selected patients with oliguric AKI that FeNa was $<1 \%$ in pre-renal AKI and $>3 \%$ in ATN. Subsequently, numerous authors described various forms of ATN with $\mathrm{FeNa}<1 \%$ and prerenal AKI with $\mathrm{FeNa}>1 \%$. One of the major reasons for reduced FeNa utility relates to the fact that various acute diseases disturb the typical tubular response and make FeNa poorly reflective of the actual cause of AKI during acute illness [38]

\section{Urine microscopy}

Small studies have shown the utility of urine microscopy in differentiating pre-renal AKI from ATN. The background is that an ischemic or nephrotoxic insult to the tubules results in apoptosis or necrosis of renal tubular epithelial cells, which are shed into the tubular lumen and excreted free or as casts. In contrast, in pre-renal AKI, urine microscopy typically shows a bland sediment or hyaline casts. However, pre-renal AKI and ATN are a spectrum and may sometimes coexist. Marcussen and colleagues [39] performed urine microscopy in 51 patients with hospital-acquired AKI who had pre-renal AKI, 'non-ATN' AKI, or ATN. They found that the number of urinary cells and granular casts correlated with the rise in serum creatinine (that is, worsening AKI). In addition, patients who needed RRT had more casts than patients who did not require RRT. In 2008, Chawla and colleagues [40] demonstrated that an 'AKI cast scoring index' based on tubular and granular casts was useful in predicting severity and non-recovery from AKI. In 2010, Perazella and colleagues [41] used urine microscopy and a modified urine sediment score to evaluate the outcome of AKI in 197 patients with pre-renal AKI or ATN. The urine sediment score was predictive of progressive AKI, need for RRT and death.

Thus, urine microscopy appears to have utility not only in differentiating AKI but also in predicting severity of AKI and outcome. Although it is inexpensive and readily available, it requires training and experience and is time consuming. The first study to compare traditional urinary biomarkers (including urine microscopy) with novel biomarkers was performed by Bagshaw and colleagues [42] in 83 septic and non-septic ICU patients with AKI. In a prospective, two-centre cohort study, they examined the utility of a urine microscopy score, urine chemistry and $\mathrm{pNGAL/uNGAL}$ in predicting worsening renal function, need for RRT, and death. The urine microscopy score was higher in septic patients with AKI, correlated with uNGAL $(\mathrm{r}=0.41 ; P=0.012)$, predicted worsening AKI and was associated with greater likelihood of RRT or death. Urine chemistry (urine sodium, FeNa) was not associated with the clinical outcomes. The authors concluded septic AKI was associated with greater urine microscopy evidence of kidney injury compared with non-septic AKI, that a higher urine microscopy score correlated with higher UNGAL and that urine microscopy and uNGAL were complementary in predicting worsening AKI in ICU patients.

In conclusion, on the basis of the current information, it appears that urine microscopy and new urine biomarkers may be used together to inform on early AKI, differentiate pre-renal AKI from ATN, and predict progressive AKI, need for RRT and death. In contrast, urine chemistry may provide some insight into differential diagnosis when used in select patients but has little role in most hospitalized patients with AKI.

\section{Urine output}

Multiple mechanisms can potentially cause a low urine output in the acutely injured kidney. Proponents argue that urine output is a valuable renal biomarker which often portends renal dysfunction in critical care patients before changes in serum creatinine $[43,44]$. In contrast, 
critics point out that urine output is affected by volume status, intrinsic levels of antidiuretic hormone, presence of obstruction, and use of diuretics, and that urine volumes can only be accurately assessed in patients with a urinary catheter.

Macedo and colleagues [45] performed hour-to-hour urine collections in a cohort of 317 critically ill surgical patients who did not have AKI, pre-existing CKD stage 5, a functioning renal transplant, liver cirrhosis or severe anaemia. Daily and cumulative fluid balance were collected between ICU admission and discharge, and admission serum creatinine was considered the reference value. Detailed fluid and diuretic administration were not recorded. The authors showed that significant renal injury was likely when patients showed persistent, consistent oliguria for 6 hours or more. They also showed an earlier detection of AKI by oliguria rather than serum creatinine. Although urine output is a criterion in both the RIFLE and AKIN classification, the data are far from robust, and despite the fact that oliguria is often a portend of a renal insult, we do not fully appreciate the significance nor the mechanisms involved in the critically ill [46].

\section{Unanswered questions}

The new biomarkers of AKI have enhanced our understanding of some of the biological and biochemical processes during AKI; however, to date their clinical utility remains unclear. It is not known whether any of the new biomarkers add anything beyond clinical evaluation and traditional renal function tests and whether they can guide clinical management and ultimately alter the outcome of patients with AKI. There is also concern that a single biomarker may not be able to fulfil all criteria as outlined in Table 1, and that it is more realistic to search for a panel of different biomarkers that, in combination, may provide the necessary data to manage patients with AKI.

Critics have also pointed out that the benefit of early diagnosis of AKI is questionable given the fact that there is no specific cure for AKI yet. That is true, but this also reflects the fact that AKI is not one disease and, as such, a specific cure is unlikely. A case could be made that early diagnosis of AKI may avoid further harm, that is, avoiding nephrotoxic drug administration or premature discharge from the emergency department or ICU.

Some studies have demonstrated that biomarkers may be elevated without overt changes in serum creatinine. In a pooled analysis of aggregate data from 10 studies including 2,322 patients, $19.2 \%$ of patients had raised NGAL levels without relevant changes in serum creatinine [47]. Mortality, length of stay in ICU and need for RRT were significantly higher in this cohort compared to patients without NGAL and creatinine rises. Whether NGAL in these circumstances is an indicator of subclinical renal injury (as suggested by the authors) or simply a marker of severity of illness remains unclear.

Despite numerous unanswered questions and uncertainties, the discovery of new biomarkers for AKI has opened the doors to a better understanding of the processes involved in AKI. More studies are necessary to improve our interpretation of biomarker values. Previous studies have concentrated on comparing the results with changes in serum creatinine, and the performance of biomarkers, including the AUC, was judged by the level of agreement. Given the well known pitfalls of serum creatinine, however, it is possible that this approach is flawed and may account for some of the inconsistent and contradictory findings in the literature. Ultimately, clinical usefulness of a set of markers will be determined not only by accuracy and reliability in prediction but also by whether that information alters therapy and whether this translates into an improvement in clinical outcomes.

The main potential for new biomarkers are: to identify the pathogenetic/pathophysiological pathways involved in the development of AKI; to function both as screening and monitoring tools for future intervention trials; and to potentially measure renal cellular injury in drug development projects.

It is likely that the biomarker profile of AKI postcardiac surgery is different from that of septic AKI or toxin-induced AKI. Patterns of AKI biomarkers may have the potential to discriminate between the various mechanisms and anatomical sites of different types of acute renal injury. In addition, clinicians are interested in knowing whether new biomarkers can be utilised to change management and outcome of AKI. Two potential study designs with incorporation of biomarkers have already been proposed [48]. In the first design, patients are randomly assigned to have the marker measured, and those with an increase are treated. In the second design, all patients have the marker measured and those with increased levels are randomly assigned to treatment or not. One such trial was performed by Endre and colleagues [49], who investigated in a double-blind placebo-controlled study whether early treatment with high dose erythropoietin could prevent the development of clinically diagnosed AKI in ICU patients. The decision to randomly assign patients was based solely on a threefold rise in urinary $\gamma$-GT and alkaline phosphatase but not on any other traditional factors. Although the study was negative and erythropoietin was not successful in preventing AKI, RRT or death, it serves as an example of how to conduct future clinical trials. In addition, biomarkers may be used as surrogate markers for response to treatment or markers of recovery from AKI, including the timing of discontinuing RRT.

Finally, early markers of nephrotoxicity have the potential to facilitate the development of safer new drugs 
[50]. They may indicate renal injury earlier than conventional methods, plus reduce the need for more invasive investigations, including renal biopsies. Following studies involving international centres and major pharmaceutical companies, the US Food and Drug Administration (FDA) and the European Medicines Agency (EMEA) have accepted multiple rodent urinary and plasma biomarkers as surrogates for renal histology for preliminary evaluation and monitoring of nephrotoxicity in drug development and monitoring during post-marketing surveillance [51].

In conclusion, the recognition that relatively trivial rises in creatinine may herald potentially disastrous sequelae for patients has led to an increased awareness of potential AKI. Hopefully, the advent of new biomarker(s) will lead to adoption of different methods of defining the kidney at risk rather than relying simply on creatinine. In clinical practice we already employ excellent biomarkers, such as highly sensitive troponin I, which has recently been shown to have an AUC of 0.96 for diagnosing acute myocardial infarction [52]. Although now fully established in clinical practice, it is important to acknowledge that it took years for measurement of troponin to become a routine clinical investigation in patients with cardiac chest pain. To date, none of the new AKI biomarkers have undergone a similar rigorous assessment but the current progress will hopefully lead to success and ultimately to improvement in patient outcomes.

\section{Abbreviations}

Y-GT, Y-glutamyl transpeptidase; ACR, albumin creatinine ratio; AKI, acute kidney injury; AKIN, Acute Kidney Injury Network; ATN, acute tubular necrosis; $A \cup C$, area under the receiver operating characteristics curve; $\mathrm{Cl}$, confidence interval; CKD, chronic kidney disease; FeNa, fractional excretion of sodium; GFR, glomerular filtration rate; IL, interleukin; KIM, kidney injury molecule; L-FABP, liver-type fatty acid-binding protein; NGAL, neutrophil gelatinaseassociated lipocalin; pNGAL, plasma neutrophil gelatinase-associated lipocalin; RIFLE, Risk - Injury - Failure - Loss - End stage renal disease; RRT, renal replacement therapy; UNGAL, urinary neutrophil gelatinase-associated lipocalin.

\section{Competing interests}

The authors declare that they have no competing interests.

\section{Author details}

'King's College London, Guy's and St Thomas' Hospital, Department of Critical Care and Nephrology, London SE1 7EH, UK. 2St George's Healthcare Trust, St George's Hospital, Department of Critical Care, London SW17 0QT, UK. ${ }^{3}$ Western Sussex Hospitals Trust, Department of Critical Care, Worthing, West Sussex, BN11 2DH, UK.

Published: 21 September 2012

\section{References}

1. Bellomo R, Ronco C, Kellum JA, Mehta RL, Palevsky P; ADQI workgroup: Acute renal failure - definition, outcome measures, animal models, fluid therapy and information technology needs: the Second International Consensus Conference of the Acute Dialysis Quality Initiative (ADQI) Group. Crit Care 2004, 8:R204-R212

2. Mehta RL, Kellum JA, Shah SV, Molitoris BA, Ronco C, Warnock DG, Levin A. Acute Kidney Injury Network: Acute Kidney Injury Network: report of an initiative to improve outcomes in acute kidney injury. Crit Care 2007, 11:R31
3. NCEPOD: Adding Insult to Injury [http://www.ncepod.org.uk/2009report1/ Downloads/AKI_summary.pdf]

4. American Society of Nephrology Renal Research Report. J Am Soc Nephrol 2005, 16:1886-1903.

5. de Geus HR, Woo JG, Wang Y, Devarajan P, Betjes MG, le Noble JL, Bakker J: Urinary NGAL measured on admission to the Intensive Care Unit accurately discriminates between sustained and transient acute kidney injury in adult critically ill patients. Nephron Extra 2011, 1:9-23.

6. Nickolas TL, Schmidt-Ott KM, Canetta P, Forster C, Singer E, Sise M, Elger A, Maarouf O, Sola-Del Valle DA, O'Rourke M, Sherman E, Lee P, Geara A, Imus P, Guddati A, Polland A, Rahman W, Elitok S, Malik N, Giglio J, El-Sayegh S, Devarajan P, Hebbar S, Saggi SJ, Hahn B, Kettritz R, Luft FC, Barasch J: Diagnostic and prognostic stratification in the emergency department using urinary biomarkers of nephron damage a multicenter prospective cohort study. J Am Coll Cardiol 2012, 59:246-255.

7. Hall IE, Coca SG, Perazella MA, Eko UU, Luciano RL, Peter PR, Han WK, Parikh CR: Risk of poor outcomes with novel and traditional biomarkers at clinical AKI diagnosis. Clin J Am Soc Nephrol 2011, 6:2740-2749.

8. Nejat M, Pickering JW, Devarajan P, Bonventre JV, Edelstein CL, Walker RJ, Endre ZH: Some biomarkers of acute kidney injury are increased in prerenal acute injury. Kidney Int 2012, 81:1254-1262.

9. Singer E, Elger A, Elitok S, Kettritz R, Nickolas TL, Barasch J, Luft FC, SchmidtOtt KM: Urinary neutrophil gelatinase-associated lipocalin distinguishes pre-renal from intrinsic renal failure and predicts outcomes. Kidney Int 2011, 80:405-414.

10. Heller F, Frischmann S, Grünbaum M, Zidek W, Westhoff TH: Urinary calprotectin and the distinction between prerenal and intrinsic acute kidney injury. Clin J Am Soc Nephrol 2011, 6:2347-2355.

11. Cruz DN, de Cal M, Garzotto F, Perazella MA, Lentini P, Corradi V, Piccinni P, Ronco C: Plasma neutrophil gelatinase-associated lipcalin is an early biomarker for acute kidney injury in an adult ICU population. Intensive Care Med 2010, 36:444-451.

12. Constantin JM, Futier E, Perbet S, Roszyk L, Lautrette A, Gillart T, Guerin R, Jabaudon M, Souweine B, Bazin JE, Sapin V: Plasma neutrophil gelatinaseassociated lipocalin is an early marker of acute kidney injury in adult critically ill patients : a prospective study. J Crit Care 2010, 25:176.e1-176.e6.

13. Endre ZH, Pickering JW, Walker RJ, Devarajan P, Edelstein CL, Bonventre JV, Frampton CM, Bennett MR, Ma Q, Sabbisetti VS, Vaidya VS, Walcher AM, Shaw GM, Henderson SJ, Nejat M, Schollum JB, George PM: Improved performance of urinary biomarkers of acute kidney injury in the critically ill by stratification for injury duration and baseline renal function. Kidney Int 2011, 79:1119-1130.

14. De Geus HR, Bakker J, Lesaffre EM, le Noble JL: Neutrophil gelatinaseassociated lipocalin at ICU admission predicts for acute kidney injury in adult patients. Am J Respir Crit Care Med 2011, 183:907-914.

15. Zhang Z, Lu B, Sheng $X$, Jin N: Cystatin C in prediction of acute kidney injury: a systemic review and meta-analysis. Am J Kidney Dis 2011, 58:356-365.

16. Royakkers AA, Korevaar JC, van Suijlen JD, Hofstra LS, Kuiper MA, Spronk PE, Schultz MJ, Bouman CS: Serum and urine cystatin C are poor biomarkers for acute kidney injury and renal replacement therapy. Intensive Care Med 2011, 37:493-501

17. Doi K, Negishi K, Ishizu T, Katagiri D, Fujita T, Matsubara T, Yahagi N, Sugaya T, Noiri E: Evaluation of new acute kidney injury biomarkers in a mixed intensive care unit. Crit Care Med 201 1, 39:2464-2469.

18. Breidthardt T, Socrates T, Drexler B, Noveanu M, Heinisch C, Arenja N, Klima T, Züsli C, Reichlin T, Potocki M, Twerenbold R, Steiger J, Mueller C: Plasma neutrophil gelatinase-associated lipocalin for the prediction of acute kidney injury in acute heart failure. Crit Care 2012, 16:R2.

19. Shapiro NI, Trzeciak S, Hollander JE, Birkhahn R, Otero R, Osborn TM, Moretti E, Nguyen HB, Gunnerson K, Milzman D, Gaieski DF, Goyal M, Cairns CB, Kupfer K, Lee SW, Rivers EP: The diagnostic accuracy of plasma neutrophil gelatinase-associated lipocalin in the prediction of acute kidney injury in emergency department patients with suspected sepsis. Ann Emerg Med 2010, 56:52-59.

20. Koyner JL, Garg AX, Coca SG, Sint K, Thiessen-Philbrook H, Patel UD, Shlipak MG, Parikh CR; TRIBE-AKI Consortium: Biomarkers predict progression of acute kidney injury after cardiac surgery. J Am Soc Nephrol 2012, 23:905-914.

21. Bell M, Granath F, Martensson J, Lofberg E, Ekbom A, Martling CR: Cystatin C is correlated with mortality in patients with and without acute kidney 
injury. Nephrol Dial Transplant 2009, 24:3096-3102.

22. Nejat M, Pickering JW, Walker RJ, Endre ZH: Rapid detection of acute kidney injury by plasma cystatin $\mathrm{C}$ in the intensive care unit. Nephrol Dial Transplant 2010, 25:3283-3289.

23. Srisawat N, Murugan R, Lee M, Kong L, Carter M, Angus DC, Kellum JA Genetic and Inflammatory Markers of Sepsis (GenIMS) Study Investigators: Plasma neutrophil gelatinase-associated lipocalin predicts recovery from acute kidney injury following community-acquired pneumonia. Kidney Int 2011, 80:545-552.

24. Hollmen ME, Kyllonen LE, Inkinen KA, Lalla ML, Merenmies J, Salmela KT: Deceased donor neutrophil gelatinase-associated lipocalin and delayed graft function after kidney transplantation: a prospective study. Crit Care 2011, 15:R121

25. Bataille A, Abbas S, Semoun O, Bourgeois É, Marie O, Bonnet F, Resche-Rigon M, Abboud I, Losser MR, Jacob L: Plasma neutrophil gelatinase-associated lipocalin in kidney transplantation and early renal function prediction. Transplantation 2011, 92:1024-1030

26. Cruz DN, De Geus HR, Bagshaw SM: Biomarker strategies to predict need for renal replacement therapy in acute kidney injury. Semin Dial 2011 24:124-131.

27. Herget-Rosenthal S, Marggraf G, Hüsing J, Göring F, Pietruck F, Janssen O, Philipp T, Kribben A: Early detection of acute renal failure by serum cystatin C. Kidney Int 2004, 66:1115-1122.

28. Perianayagam MC, Seabra VF, Tighiouart H, Liangos O, Jaber BL: Serum cystatin C for prediction of dialysis requirement or death in acute kidney injury: a comparative study. Am J Kidney Dis 2009, 54:1025-1033.

29. Liangos O, Perianayagam MC, Vaidya VS, Han WK, Wald R, Tighiouart $H$, MacKinnon RW, Li L, Balakrishnan VS, Pereira BJ, Bonventre JV, Jaber BL: Urinary N-acetyl-beta-(D)-glucosaminidase activity and kidney injury molecule-1 level are associated with adverse outcomes in acute renal failure. J Am Soc Nephrol 2007, 18:904-912

30. Lameire NH, Vanholder RC, Van Biesen WA: How to use biomarkers efficiently in acute kidney injury. Kidney Int 2011, 79:1047-1050.

31. Nickolas TL, O'Rourke MJ, Yang J, Sise ME, Canetta PA, Barasch N, Buchen C, Khan F, Mori K, Giglio J, Devarajan P, Barasch J: Sensitivity and specificity of a single emergency department measurement of urinary neutrophil gelatinase-associated lipocalin for diagnosing acute kidney injury. Ann Intern Med 2008, 148:810-819.

32. Cullen MR, Murray PT, Fitzgibbon MC: Establishment of a reference interval for urinary neutrophil gelatinase-associated lipocalin. Ann Clin Biochem 2012, 49:190-193.

33. Mcllroy DR, Wagener G, Lee HT: Neutrophil gelatinase-associated lipocalin and acute kidney injury after cardiac surgery: the effect of baseline renal function on diagnostic performance. Clin J Am Soc Nephrol 2010, 5:211-219.

34. Waikar SS, Sabbisetti VS, Bonventre JV: Normalization of urinary biomarkers to creatinine during changes in glomerular filtration rate. Kidney Int 2010, 78:486-494

35. Goldstein SL: Urinary kidney injury biomarkers and urine creatinine normalization: a false premise or not? Kidney Int 2010, 78:433-435.

36. Siew ED, Ware LB, GebretsadikT, Shintani A, Moons KG, Wickersham N, Bossert F, Ikizler TA: Urine neutrophil gelatinase-associated lipocalin moderately predicts acute kidney injury in critically ill adults. J Am SoC Nephrol 2009, 20:1823-1832.

37. Espinel $\mathrm{CH}$ : The FENa test. Use in the differential diagnosis of acute renal failure. JAMA 1976, 236:579-581.

38. Perazella MA, Coca SG: Traditional urinary biomarkers in the assessment of hospital-acquired AKI. Clin J Am Soc Nephrol 2012, 7:167-174.

39. Marcussen N, Schumann J, Campbell P, Kjellstrand C: Cytodiagnostic urinalysis is very useful in the differential diagnosis of acute renal failure and can predict the severity. Ren Fail 1995, 17:721-729.

40. Chawla LS, Dommu A, Berger A, Shih S, Patel SS: Urinary sediment cast scoring index for acute kidney injury: A pilot study. Nephron Clin Pract 2008 110:c145-c150.

41. Perazella MA, Coca SG, Hall IE, Iyanam U, Koraishy M, Parikh CR: Urine microscopy is associated with severity and worsening of acute kidney injury in hospitalized patients. Clin J Am Soc Nephrol 2010, 5:402-408.

42. Bagshaw SM, Haase M, Haase-Fielitz A, Bennett M, Devarajan P, Bellomo R: A prospective evaluation of urine microscopy in septic and non-septic acute kidney injury. Nephrol Dial Transplant 2012, 27:582-588.

43. Molitoris BA, Levin A, Warnock DG, Joannidis M, Mehta RL, Kellum JA, Ronco C. Shah S: Acute Kidney Injury Network: Improving outcomes from acute kidney injury. J Am Soc Nephrol 2007, 18:1992-1993.

44. Cerda J: Oliguria: an earlier and accurate biomarker of acute kidney injury? Kidney Int 2011, 80:699-701

45. Macedo E, Malhotra R, Bouchard J, Wynn SK, Mehta RL: Oliguria is an early predictor of higher mortality in critically ill patients. Kidney Int 2011 , 80:760-767.

46. Legrand $M$, Payen D: Understanding urine output in critically ill patients. Ann Intensive Care 2011, 1:13.

47. Haase M, Devarajan P, Haase-Fielitz A, Bellomo R, Cruz DN, Wagener G, Krawczeski CD, Koyner JL, Murray P, Zappitelli M, Goldstein SL, Makris K, Ronco C, Martensson J, Martling CR, Venge P, Siew E, Ware LB, Ikizler TA, Mertens PR: The outcome of neutrophil gelatinase-associated lipocalinpositive subclinical acute kidney injury: a multicenter pooled analysis of prospective studies. J Am Coll Cardiol 2011, 57:1752-1761.

48. Parikh CR, Garg AX: Acute kidney injury: better biomarkers and beyond. Kidney Int 2008, 73:801-803.

49. Endre ZH, Walker RJ, Pickering JW, Shaw GM, Frampton CM, Henderson SJ, Hutchison R, Mehrtens JE, Robinson JM, Schollum JB, Westhuyzen J, Celi LA, McGinley RJ, Campbell IJ, George PM: Early intervention with erythropoietin does not affect the outcome of acute kidney injury (the EARLYARF trial). Kidney Int 2010, 77:1020-1030

50. Waring WS, Moonie A: Earlier recognition of nephrotoxicity using novel biomarkers of acute kidney injury. Clin Toxicol 2011, 49:720-728.

51. Dieterle F, Sistare F, Goodsaid F, Papaluca M, Ozer JS, Webb CP, Baer W, Senagore A, Schipper MJ, Vonderscher J, Sultana S, Gerhold DL, Phillips JA Maurer G, Carl K, Laurie D, Harpur E, Sonee M, Ennulat D, Holder D, AndrewsCleavenger D, Gu YZ, Thompson KL, Goering PL, Vidal JM, Abadie E, Maciulaitis $R$, Jacobson-Kram D, Defelice AF, Hausner EA, et al:: Renal biomarker qualification submission: A dialog between the FDA-EMEA and Predictive Safety Testing Consortium. Nat Biotechnol 2010, 28:455-462.

52. Keller T, Zeller T, Ojeda F, Tzikas S, Lillpopp L, Sinning C, Wild P, Genth-Zotz S, Warnholtz A, Giannitsis E, Möckel M, Bickel C, Peetz D, Lackner K, Baldus S, Münzel T, Blankenberg S: Serial changes in highly sensitive troponin I assay and early diagnosis of myocardial infarction. JAMA 2011, 306:2684-2693.

53. Liangos O, Tighiouart $\mathrm{H}$, Perianayagam MC, Kolyada A, Han WK, Wald R, Bonventre JV, Jaber BL: Comparative analysis of urinary biomarkers for early detection of acute kidney injury following cardiopulmonary bypass. Biomarkers 2009, 14:423-431.

54. Haase-Fielitz A, Bellomo R, Devarajan P, Story D, Matalanis G, Dragun D, Haase M: Novel and conventional serum biomarkers predicting acute kidney injury in adult cardiac surgery - a prospective cohort study. Crit Care Med 2009, 37:553-560.

55. Haase M, Bellomo R, Devarajan P, Ma Q, Bennett MR, Möckel M, Matalanis G, Dragun D, Haase-Fielitz A: Novel biomarkers predict the severity of acute kidney injury after cardiac surgery in adults. Ann Thorac Surg 2009, 88:124-130.

56. Koyner UL, Bennett MR, Worcester EM, Ma Q, Raman J, Jeevanandam V, Kasza $\mathrm{KE}, \mathrm{O}^{\prime}$ Connor MF, Konczal DJ, Trevino S, Devarajan P, Murray PT: Urinary cystatin $\mathrm{C}$ as an early biomarker of acute kidney injury following adult cardiothoracic surgery. Kidney Int 2008, 74:1059-1069

57. Tuladhar SM, Puntmann VO, Soni M, Punjabi PP, Bogle RG: Rapid detection of acute kidney injury following cardiopulmonary bypass. J Cardiovasc Pharmacol 2009, 53:261-266.

58. Han WK, Wagener G, Zhu Y, Wang S, Lee HT: Urinary biomarkers in the early detection of acute kidney injury after cardiac surgery. Clin J Am Soc Nephrol 2009, 4:873-882.

59. Xin C, Yulong X, Yu C, Changchun C, Feng Z, Xinwei M: Urine neutrophil gelatinase-associated lipocalin and interleukin-18 predict acute kidney injury after cardiac surgery. Ren Fail 2008, 30:904-913.

60. Wagener G, Gubitosa G, Wang S, Borregaard N, Kim M, Lee HT: Urinary neutrophil gelatinase-associated lipocalin and acute kidney injury after cardiac surgery. Am J Kidney Dis 2008, 52:425-433.

61. Wagener G, Jan M, Kim M, Mori K, Barasch JM, Sladen RN, Lee HT: Association between increases in urinary neutrophil gelatinase-associated lipocalin and acute renal dysfunction after adult cardiac surgery. Anesthesiology 2006, 105:485-491.

62. Haase-Fielitz A, Bellomo R, Devarajan P, Bennett M, Story D, Matalanis G, Frei $U$, Dragun $D$, Haase M: The predictive performance of plasma neutrophil gelatinase-associated lipocalin (NGAL) increases with grade of acute kidney injury. Nephrol Dial Transplant 2009, 24:3349-3354.

63. Parikh CR, Coca SG, Thiessen-Philbrook H, Shlipak MG, Koyner UL, Wang Z 
Edelstein CL, Devarajan P, Patel UD, Zappitelli M, Krawczeski CD, Passik CS, Swaminathan M, Garg AX;TRIBE-AKI Consortium: Postoperative biomarkers predict acute kidney injury and poor outcomes after adult cardiac surgery. J Am Soc Nephrol 2011, 22:1748-1757.

64. Ling W, Zhaohui N, Ben H, Leyi G, Jianping L, Huili D, Jiaqi Q: Urinary IL-18 and NGAL as early predictive biomarkers in contrast-induced nephropathy after coronary angiography. Nephron 2008, 108:c176-181.

65. Rickli H, Benou K, Ammann P, Fehr T, Brunner-La Rocca HP, Petridis H, Riesen W, Wüthrich RP: Time course of serial cystatin C levels in comparison with serum creatinine after application of radiocontrast media. Clin Nephrol 2004, 61:98-102

66. Bagshaw SM, Bennett M, Haase M, Haase-Fielitz A, Egi M, Morimatsu H, D'amico G, Goldsmith D, Devarajan P, Bellomo R: Plasma and urine neutrophil gelatinase-associated lipocalin in septic versus non-septic acute kidney injury in critical illness. Intensive Care Med 2010, 36:452-461.

67. Mårtensson J, Bell M, Oldner A, Xu S, Venge P, Martling CR: Neutrophil gelatinase-associated lipocalin in adult septic patients with and without acute kidney injury. Intensive Care Med 2010, 36:1333-1340.

68. Makris K, Markou N, Evodia E, Dimopoulou E, Drakopoulos I, Ntetsika K, Rizos D, Baltopoulos G, Haliassos A: Urinary neutrophil gelatinase-associated lipocalin (NGAL) as an early marker of acute kidney injury in critically ill multiple trauma patients. Clin Chem Lab Med 2009, 47:79-82.

69. Hall IE, Yarlagadda SG, Coca SG, Wang Z, Doshi M, Devarajan P, Han WK, Marcus RJ, Parikh CR: IL-18 and urinary NGAL predict dialysis and graft recovery after kidney transplantation. J Am Soc Nephrol 2010, 21:189-197.

70. Yang HN, Boo CS, Kim MG, Jo SK, Cho WY, Kim HK: Urine neutrophil gelatinase-associated lipocalin: an independent predictor of adverse outcomes in acute kidney injury. Am J Nephrol 2010, 31:501-509.

71. Kümpers $P$, Hafer C, Lukasz A, Lichtinghagen R, Brand K, Fliser D, FaulhaberWalter R, Kielstein JT: Serum neutrophil gelatinase-associated lipocalin at inception of renal replacement therapy predicts survival in critically ill patients with acute kidney injury. Crit Care 2010, 14:R9

72. Bolignano D, Lacquaniti A, Coppolino G, Campo S, Arena A, Buemi M: Neutrophil gelatinase-associated lipocalin reflects the severity of renal impairment in subjects affected by chronic kidney disease. Kidney Blood Press Res 2008, 31:255-258.

73. Villa P, Jimenez M, Soriano MC, Manzanares J, Casasnovas P: Serum cystatin concentration as a marker of acute renal dysfunction in critically ill patients. Crit Care 2005, 9:R139-143

74. Wald R, Liangos O, Perianayagam MC, Kolyada A, Herget-Rosenthal S, Mazer $C D$, Jaber BL: Plasma cystatin C and acute kidney injury after cardiopulmonary bypass. Clin J Am Soc Nephrol 2010, 5:1373-1379.

75. Ling $Q, X u X, L i J$ J, Chen J, Shen JW, Zheng SS: Alternative definition of acute kidney injury following liver transplantation: based on serum creatinine and cystatin C levels. Transplant Proc 2007, 39:3257-3260.

76. Bachorzewska-Gajewska H, Malyszko J, Sitniewska E, Malyszko JS, Pawlak K, Mysliwiec M, Lawnicki S, Szmitkowski M, Dobrzycki S: Could neutrophil gelatinase-associated lipocalin and cystatin C predict the development of contrast-induced nephropathy after percutaneous coronary interventions in patients with stable angina and normal serum creatinine values? Kidney Blood Press Res 2007, 30:408-415.

77. Vaidya VS, Waikar SS, Ferguson MA, Collings FB, Sunderland K, Gioules C, Bradwin G, Matsouaka R, Betensky RA, Curhan GC, Bonventre JV: Urinary biomarkers for sensitive and specific detection of acute kidney injury in humans. Clin Trans/ Sci 2008, 1:200-208.
78. Herget-Rosenthal S, Poppen D, Hüsing J, Marggraf G, Pietruck F, Jakob HG, Philipp T, Kribben A: Prognostic value of tubular proteinuria and enzymuria in nonoliguric acute tubular necrosis. Clin Chem 2004, 50:552-558.

79. Daniel JP, Chantrel F, Offner M, Moulin B, Hanndedouche T: Comparison of cystatin C, creatinine and creatinine clearance versus GFR for detection of renal failure in renal transplant patients. Ren Fail 2004, 26:253-257.

80. Ahlstrom A, Tailgren M, Peltonen S, Pettila V: Evolution and predictive power of serum cystatin C in acute renal failure. Clin Nephrol 2004, 62:344-350.

81. Parikh CR, Jani A, Mishra J, Ma Q, Kelly C, Barasch J, Edelstein CL, Devarajan P: Urine NGAL and IL-18 are predictive biomarkers for delayed graft function following kidney transplantation. Am J Transplant 2006, 6:1639-1645.

82. Parikh CR, Mishra J, Thiessen-Philbrook H, Dursun B, Ma Q, Kelly C, Dent C, Devarajan P, Edelstein CL: Urinary IL-18 is an early predictive biomarker of acute kidney after cardiac surgery. Kidney Int 2006, 70:199-203.

83. Parikh CR, Abraham E, Ancukiewicz M, Edelstein CL: Urine IL-18 is an early diagnostic marker for acute kidney injury and predicts mortality in the intensive care unit. J Am Soc Nephrol 2005, 16:3046-3052.

84. Han WK, Waikar SS, Johnson A, Betensky RA, Dent CL, Devarajan P, Bonventre $\mathrm{JV}$ : Urinary biomarkers in the early diagnosis of acute kidney injury. Kidney Int 2008, 73:863-869.

85. Han WK, Wagener G, Zhu Y, Wang S, Lee HT: Urinary biomarkers in the early detection of acute kidney injury after cardiac surgery. Clin J Am Soc Nephrol 2009, 4:873-882

86. Metzger J, Kirsch T, Schiffer E, Ulger P, Mentes E, Brand K, Weissinger EM, Haubitz M, Mischak H, Herget-Rosenthal S: Urinary excretion of twenty peptides forms an early and accurate diagnostic pattern of acute kidney injury. Kidney Int 2010, 78:1252-1262.

87. Ferguson MA, Vaidya VS, Waikar SS, Collings FB, Sunderland KE, Gioules $C J$, Bonventre JV: Urinary liver-type fatty acid-binding protein predicts adverse outcomes in acute kidney injury. Kidney Int 2010, 77:708-714.

88. Nakamura T, Sugaya T, Node K, Ueda Y, Koide H: Urinary excretion of livertype fatty acid-binding protein in contrast medium-induced nephropathy. Am J Kidney Dis 2006, 47:439-444.

89. McMahon BA, Koyner JL, Murray PT: Urinary glutathione S-transferases in the pathogenesis and diagnostic evaluation of acute kidney injury following cardiac surgery: a critical review. Curr Opin Crit Care 2010, 16:550-555.

90. Ramesh G, Krawczeski CD, Woo JG, Wang Y, Devarajan P: Urinary netrin-1 is an early predictive biomarker of acute kidney injury after cardiac surgery. Clin J Am Soc Nephrol 2010, 5:395-401.

91. Prowle JR, Ostland V, Calzavacca P, Licari E, Ligabo EV, Echeverri JE, Bagshaw SM, Haase-Fielitz A, Haase M, Westerman M, Bellomo R: Greater increase in urinary hepcidin predicts protection from acute kidney injury after cardiopulmonary bypass. Nephrol Dial Transplant 2012, 27:595-602.

doi:10.1186/cc11380

Cite this article as: Ostermann M, et al:: Clinical review: Biomarkers of acute kidney injury: where are we now? Critical Care 2012, 16:233. 